\title{
NEAR-BOTTOM FLOW CHARACTERISTICS OF CURRENTS AT ARBITRARY ANGLE TO 2D RIPPLES
}

\begin{abstract}
Ole Secher Madsen ${ }^{1}$, Arlendenovega Satria Negara ${ }^{2}$, Kian Yew Lim ${ }^{3}$, and Hin Fatt Cheong ${ }^{4}$
Experimental results for near-bottom current velocity profiles for flows over artificial, definitely 2D ripples made of $1.5 \mathrm{~cm}$ high aluminum angle-profile spaced at $10 \mathrm{~cm}$ intervals are obtained for the following cases: (i) current alone perpendicular to ripples; (ii) current alone parallel to ripples; (iii) combined orthogonal wave-current flows for current parallel to ripples; and (iv) current alone at an angle of $30^{\circ}$ to the ripple axis. The velocity profiles are analyzed by the log-profile method, and show the roughness experienced by the current to increase as the angle between ripple and current direction increases, i.e. demonstrating convincingly the reality of the concept of a direction-dependent roughness for flows over a $2 \mathrm{D}$ rippled bottom. Roughness experienced by the velocity component perpendicular to the ripples is, however, found to be independent of the direction of the mainstream flow relative to that of the ripples, and the different roughness experienced by the perpendicular and parallel velocity components gives rise to a turning of the current velocity vector to become increasingly aligned with the ripple crests as the bottom is approached from above. Implications of this feature, in terms of net sediment transport direction in combined wave-current flows in inner-shelf coastal waters, is discussed.
\end{abstract}

Keywords: flow over ripples; bottom roughness; wave-current interaction; boundary layer flows over $2 D$ roughness elements

\section{INTRODUCTION}

In coastal waters currents are predominantly shore-parallel and therefore near-perpendicular to incoming waves. Combined near-orthogonal wave-current flows are therefore of more fundamental importance to hydrodynamic and sediment transport processes in coastal waters than combined codirectional wave-current flows. However, owing to difficulties in establishing flows of the former type in the laboratory setting, there are relatively few experimental results available on orthogonal wavecurrent flows, whereas there is a relative wealth of experimental data on co-directional wave-current flows. With this as their motivation, Madsen, Kularatne and Cheong (2008), hereafter referred to as MKC08, presented experimental results on bottom roughness experienced by currents alone and perpendicular to waves over a movable bottom consisting of $0.18 \mathrm{~mm}$ diameter quartz sand. For a current alone over a wave-rippled bed MKC08 found that the movable bottom roughness experienced by the current was of the order of the ripple height, whereas a movable bottom roughness of the order four times the ripple height, in combination with the modified wave-current interaction model by Madsen (1994), successfully predicted the observed current velocity profile in the presence of perpendicular waves. Both of these experimentally obtained movable bottom roughness values are surprisingly large when considering the current direction relative to the ripple axis orientation (nearparallel), and were tentatively explained as a result of the wave-generated ripple-pattern exhibiting some 3D features, e.g. ripple crests were not "exactly" straight and aligned with the wave crests (see Figure 4 of MKC08).

To address this unresolved problem of the surprisingly large movable bed roughness obtained in MKC08, we undertook an idealized experimental study of the nature of near-bottom flow characteristics of currents over an artificially, and hence strictly 2D, rippled bottom. Except for a preliminary experiment, which was conducted in a flume, the bulk of the experiments were conducted in the same experimental facility (the wave basin in the Hydraulics Laboratory at the National University of Singapore (NUS) using the same experimental and data analysis procedures as described in details by $\mathrm{MKC} 08$. All experiments presented in this paper were all conducted for flows over an artificially $2 \mathrm{D}$ rippled bed consisting of $1.5 \mathrm{~cm}$ high right-angle aluminum profiles (bars), spot-welded with silicone adhesive to the actual bottom, and spaced at $10 \mathrm{~cm}$ intervals.

Experimental results will be presented and discussed in subsequent sections for the following cases:

${ }^{1}$ Donald and Martha Harleman Professor, Department of Civil and Environmental Engineering, Ralph M. Parsons Laboratory, 48-216C, MIT, Cambridge, MA 02139, USA. Email: osm@mit.edu

${ }^{2}$ Formerly: Graduate student, Department of Civil Engineering, National University of Singapore; Now: Staff of Planning, Bengawan Solo River Basin Agency, Jalan Solo-Kartasura Km. 7, PO Box 267, Surakarta 57102, Indonesia. Email: satrianegara@gmail.com

${ }^{3}$ Graduate Student, Department of Civil Engineering, National University of Singapore, 1 Engineering Drive 2, EW1 03-01A, Singapore 117576. Email: limkianyew@nus.edu.sg

${ }^{4}$ Professor, Department of Civil Engineering, National University of Singapore, 1 Engineering Drive 2, E1A 07-03,

Singapore 117576. Email: cvechf@nus.edu.sg 

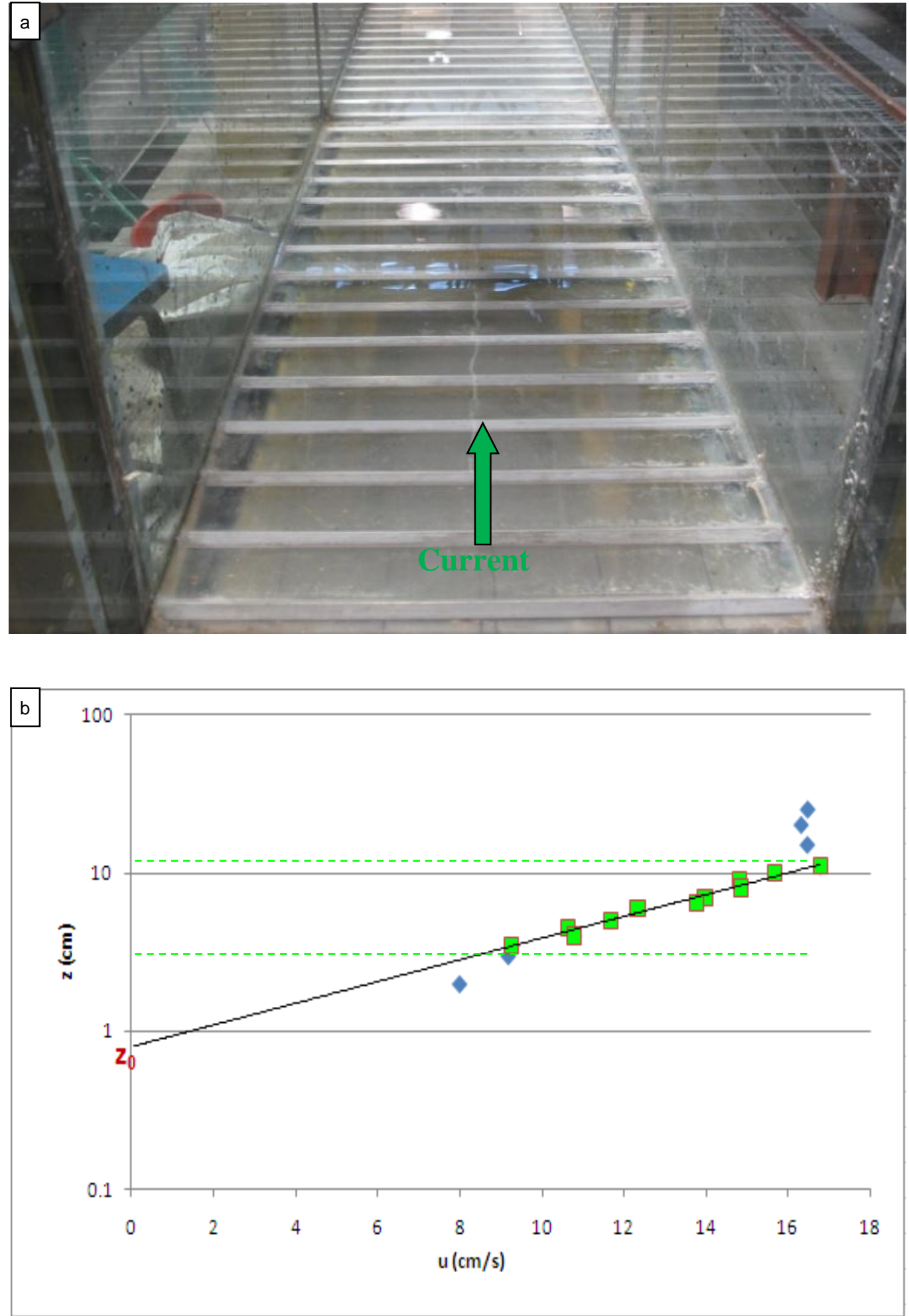

Figure 1. Experiments for current alone perpendicular to ripples (Case 1). (a) Photo of experimental set-up in flume at NUS (b) Semi-log plot of typical velocity profile; only data within z-range indicated by dashed lines (square symbols) used to obtain best fit log-profile. 
1. Current alone over artificial ripples perpendicular to current-direction

2. Current alone over artificial ripples parallel to current-direction

3. Combined orthogonal waves and currents over artificial ripples parallel to current-direction

4. Current alone over artificial ripples with ripple-axis at $30^{\circ}$ to current-direction

The last section of the paper will present our conclusions and some implications of our findings.

\section{EXPERIMENTAL RESULTS}

\section{Case 1: Current alone perpendicular to ripples}

A preliminary, base-line, experiment for a current alone perpendicular to the artificial ripples was conducted in a flume at the Hydraulics Laboratory at NUS. The experimental set-up is shown in Figure $1 \mathrm{a}$, and the current velocity profile measured at the centerline of the flume a distance of $3 \mathrm{~m}$ downstream of the start of the ripples is shown in semi-log form in Figure 1b. As explained in MKC08, only velocity data well within the developing current boundary layer, of estimated thickness $\delta_{c}$, and far enough from the bottom for the flow to be unaffected by the proximity of individual roughness elements, are used in the log-profile analysis to obtain experimental values for the equivalent Nikuradse sand grain roughness, $k_{N}$. This range, $\sim 2 \eta=3.0 \mathrm{~cm}<\mathrm{z}<\sim(2 / 3) \delta_{\mathrm{c}}$, where $\eta$ denotes the ripple height, is clearly identified in Figure 1b. Based on analysis of $\sim 20$ velocity profiles obtained at different locations of the ripple-covered flume bottom (see Negara (2010) for details) we obtain

$$
k_{N c \perp}=30 z_{0}=18.7 \pm 3.3 \mathrm{~cm}
$$

which is in excellent agreement with the value of $20.9 \mathrm{~cm}$ obtained by Mathisen and Madsen (1996) for the same artificial ripple configuration as used here.

\section{Case 2: Current alone parallel to ripples}

A series of experiments for pure currents aligned with the axis of the artificial ripples was performed in the NUS Hydraulics Laboratory's wave basin, modified to accommodate combined orthogonal wave-current flows as described in MKC08. The experimental set-up is visualized in Figure 2a, in which the current is coming towards the camera and waves will enter from the right in combined orthogonal wave-current experiments. Also seen in the photo are the wave-guides that help confine the current to "current channel" they form, while interfering minimally with the waves when these are present. Here we are dealing with a pure current, whereas combined wave-current flows will be presented as Case 3 in the next section.

Following MKC08, care was taken to ascertain that the set-up produced a current that was uniform across the width $(2 \mathrm{~m})$ and depth $(0.40 \mathrm{~m})$ of the current channel, as it entered the basin through a honeycomb filter made from $50 \mathrm{~cm}$ long, $5 \mathrm{~cm}$ diameter PVC pipes. Also, in accordance with the experimental protocol of MKC08, the region of near-uniform flow conditions was determined. This region, extending from 3.5 to $5.0 \mathrm{~m}$ from the current inlet and $25 \mathrm{~cm}$ to either side of the centerline of the current channel, is indicated in Figure $2 \mathrm{a}$, and only velocity data obtained within this region were analyzed.

A typical semi-log plot of the measured current velocity profile is shown in Figure $2 b$, and the logprofile analysis of several $(\sim 40)$ such measurements, all obtained within the near-uniform flow region with roughly half being located directly above ripple crests, resulted in an experimental bottom roughness for a current parallel to the artificial ripple axis

$$
k_{N c \|}=30 z_{0}=0.025 \mathrm{~cm} \stackrel{\times}{\div} 3
$$

The extreme uncertainty in the roughness estimate obtained for this case, a factor of 3 , is not surprising. As indicated in Figure 2b, only data from $z>1.5 \mathrm{~cm}(z=0$ at the concrete bottom) were used in the log-profile analysis. The extrapolation of the best fit straight line to the data above $z \sim 1.5$ $\mathrm{cm}$ to $u=0$ to get $z_{0} \sim 0.001 \mathrm{~cm}$ therefore covers several decades and the experimental value obtained for $z_{0}$ is therefore extremely sensitive to even minor slope changes of this line. In addition, local irregularities in the concrete bottom elevation, i.e. the actual location of $z=0$, could be a factor contributing to the uncertainty in our roughness estimate given by (2).

In addition to $z_{0}$-values, the $\log$-profile analysis also provides experimental values for the shear velocity, $u_{*_{c}}$. For this set of experiments we obtained $u_{*_{c}}=0.54 \pm 0.02 \mathrm{~cm} / \mathrm{s}$ [note the small 

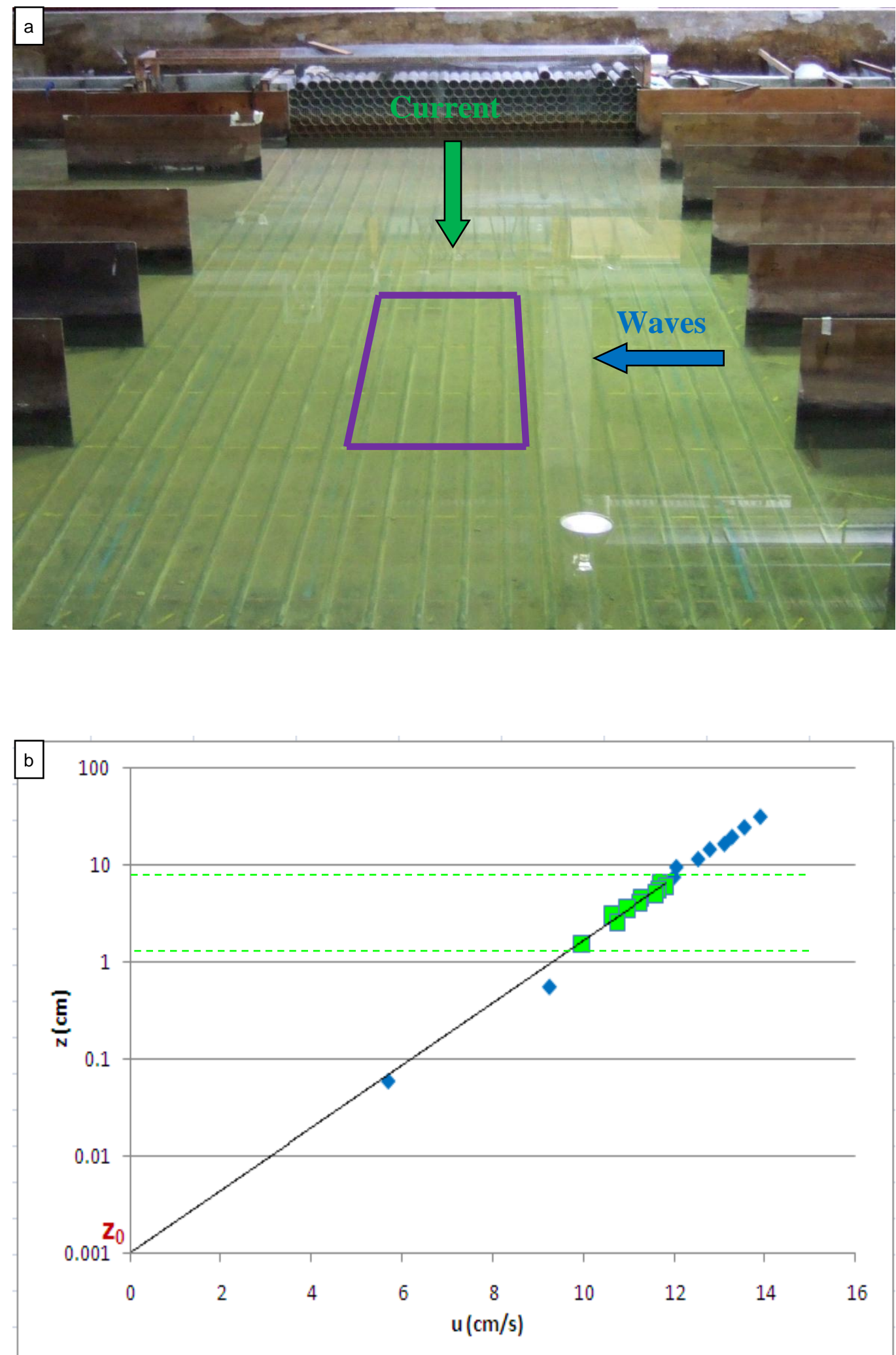

Figure 2. Experiments for current alone parallel to ripples (Case 2). (a) Photo of experimental set-up for current alone (Case 2) and in the presence of orthogonal waves (Case 3) in wave basin at NUS with outline of near-uniform flow region (b) Semi-log plot of typical velocity profile for current alone; only data within $z$ range indicated by dashed lines (square symbols) used to obtain best fit log-profile. 
uncertainty in $u_{*}$, which represents the uncertainty in the slope of the best fit line, and the resulting uncertainty of the factor of 3 for $z_{0}$ ]. Assuming smooth turbulent flow, and taking $u_{*_{c}}=0.54 \mathrm{~cm} / \mathrm{s}$, the corresponding bottom roughness for a smooth bottom is given by, e.g. Schlichting (1960),

$$
k_{N c \text {,smooth }}=30 \frac{v}{9 u_{* c}}=0.05 \mathrm{~cm}
$$

Thus, despite the uncertainty in our experimental value for the bottom roughness experienced by a current parallel to the ripple axis, we can with confidence say that this type of flow, to "leading order", does not experience a bottom roughness related to the presence of the ripples.

\section{Case 3: Combined orthogonal wave-current flow with current parallel to ripples}

In this case a periodic wave (height $=H=10 \mathrm{~cm}$, period $=T=1.5 \mathrm{~s}$ ) was added to the current condition of Case 2 (see Figure 2a for experimental set-up). By control of the current outflow, by adjusting the tailgate elevation in the outflow channel, the current discharge, $Q$, as well as the water depth $(h=0.40 \mathrm{~m})$ were maintained the same as for the current alone (Case 2). This wave condition corresponds to a near-bottom wave orbital velocity amplitude of $u_{b m}=18.8 \mathrm{~cm} / \mathrm{s}$, which is comparable to the nominal average current velocity $U_{c}=Q /(0.4 \cdot 2.0)=13.9 \mathrm{~cm} / \mathrm{s}$.

A typical semi-log plot of the current velocity profile, obtained from 3-minute averages of the recorded velocity, $u(t)$, in the direction of the current channel, i.e. parallel to the ripples, is shown in Figure 3a. As seen from this plot data from $z<\sim 6 \mathrm{~cm}$ are excluded from the log-profile analysis of the current profile. This is because data from $z<\sim 6 \mathrm{~cm}$ fall within the wave bottom boundary layer, $\delta_{w} \sim$ $6 \mathrm{~cm}$, estimated from Madsen's (1994) modified wave boundary layer theory. Since the wave orbital velocity is perpendicular to the ripples, the bottom roughness was taken as $k_{N}=18.7 \mathrm{~cm}$, i.e. as given by (1), to obtain this estimate for $\delta_{w}$. Log-profile analysis of $\sim 40$ current velocity profiles, all obtained within the near-uniform flow region of the current channel (Figure 2a), gives values for the "apparent" bottom roughness, $k_{N a}$, which is the enhanced bottom roughness experienced by the current in the presence of waves,

$$
k_{N a}=30 z_{0 a}=14.6 \pm 3.7 \mathrm{~cm}
$$

With this value of the "apparent" bottom roughness, the associated estimate of the current shear velocity, $u_{*_{c}}=1.32 \pm 0.10 \mathrm{~cm} / \mathrm{s}$, and the characteristics of the orthogonal near-bottom wave orbital velocity, $u_{b m}=18.8 \mathrm{~cm} / \mathrm{s}$ and $T=1.5 \mathrm{~s}$, Madsen's (1994) modified wave-current interaction model (See Appendix in MKC08) can be used to obtain values for the "physical" bottom roughness,

$$
k_{N c w \|}=30 z_{0}=1.58 \pm 0.41 \mathrm{~cm}
$$

The Madsen (1994) modified wave-current boundary layer model uses as input the observed current velocity profile for $z>\delta_{w}$, but predicts its continuation into the wave-current boundary layer, i.e. for $z<\delta_{w}$. This theoretical prediction of the current velocity for $z<\delta_{w}$ is shown in Figure 3a, and it is gratifying to notice that this prediction is in fair agreement with the experimentally obtained current velocities for $z<\delta_{w} \sim 6 \mathrm{~cm}$.

The estimate of the physical bottom roughness given by (5) is the relevant roughness to compare with roughness values obtained for currents alone over the same ripple configuration. Thus, we see that $k_{N c w \|}=1.58 \mathrm{~cm}$ is much smaller than the roughness experienced by a current perpendicular to the ripples, $k_{N c w \perp}=18.7 \mathrm{~cm}$ given by (1), but also far greater than the roughness obtained for a current parallel to the ripples, $0.025 \mathrm{~cm}$ as given by (2). The former observation is contradicting the result obtained in MKC08 for combined orthogonal wave-current flows over a naturally rippled bed. In MCK08 the physical bottom roughness that reproduced the observed current velocity profile characteristics above the wave boundary layer for near-identical wave and current conditions to those in the present study was found to be "the same" as the roughness experienced by a current alone in the direction perpendicular to wave-generated ripples on a movable bed. Since the present experiments were conducted with strictly 2D "ripples" whereas the movable bed ripples in the MKC08 experiments (see MKC08, Figure 4) exhibited some 3D features, the present results support the conjecture of MKC08 that their finding of direction-independent roughness was a result of three-dimensionality of their movable bed ripples. On the other hand, the drag forces associated with the wave motion perpendicular to the artificial ripples in the present experiments have no component in the direction of 

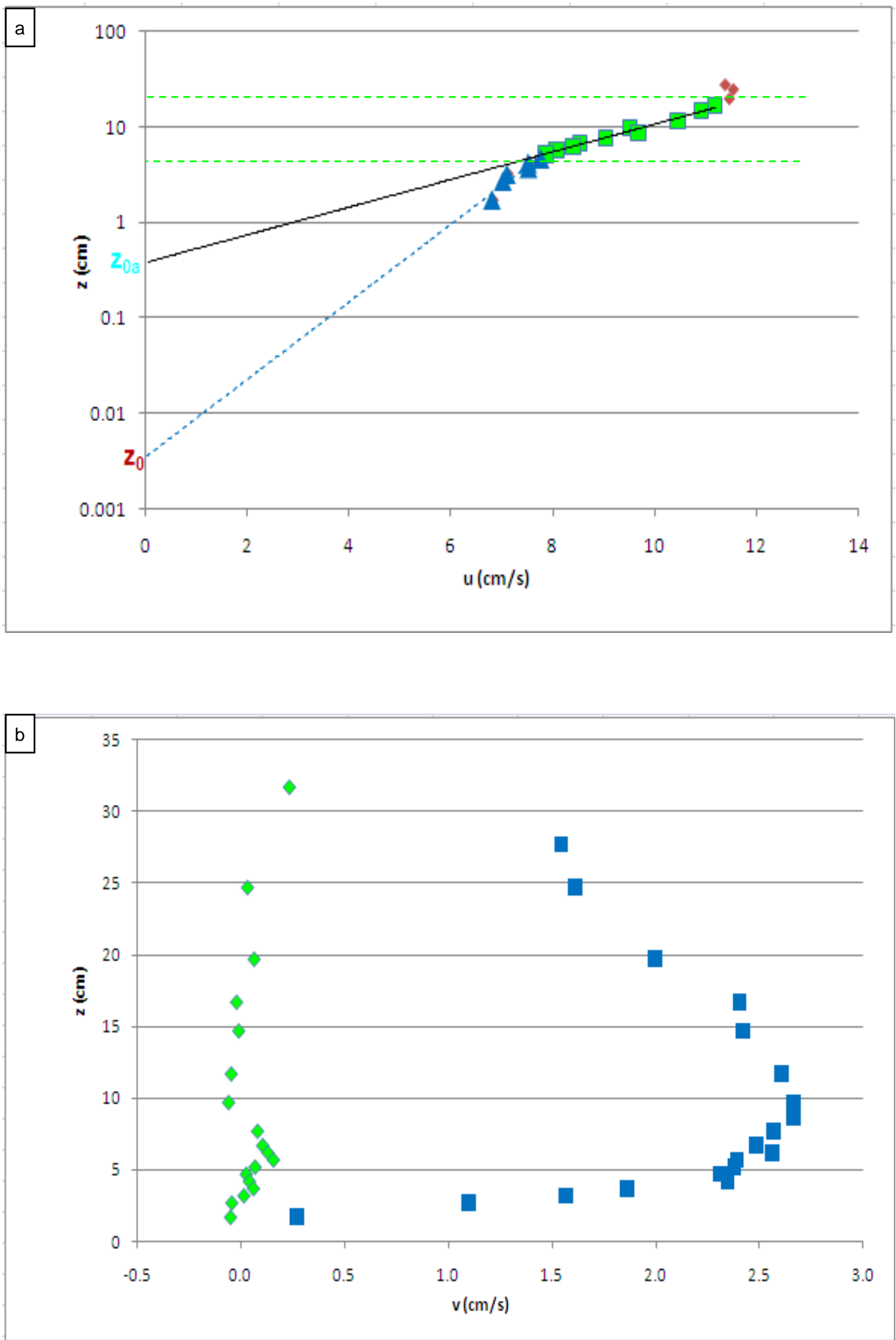

Figure 3. Experiments for current alone parallel to ripples in the presence of orthogonal waves (Case 3). (a) Semi-log plot of current velocity profile; only data within z-range indicated by dashed lines (square symbols) used to obtain best fit log-profile (full line), dashed line is the current profile inside the wave boundary layer predicted by Madsen's (1994) modified wave-current model (b) Current velocity perpendicular to ripples in the absence (diamonds) and presence (squares) of waves. 

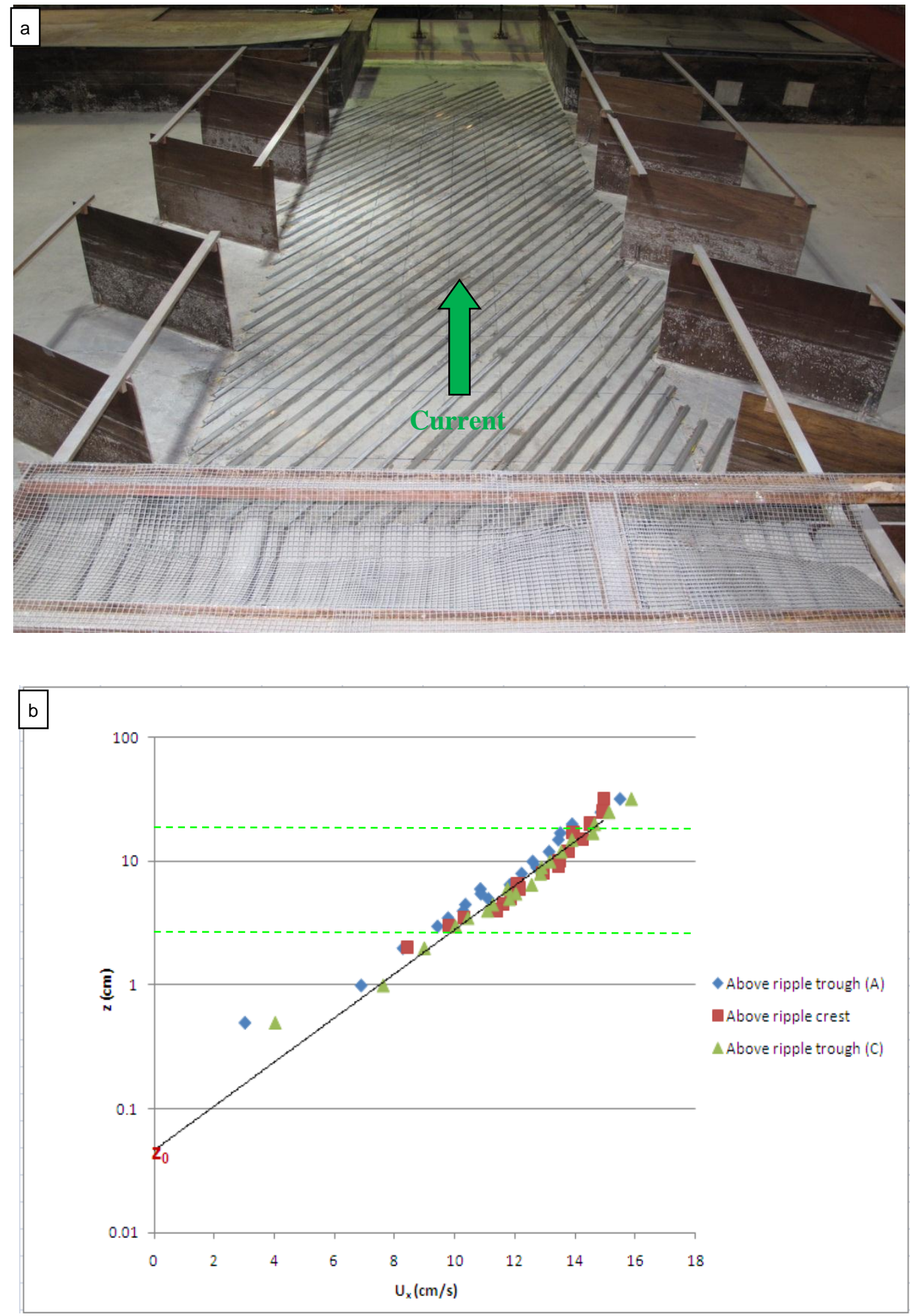

Figure 4. Experiments for current alone at $30^{\circ}$ to ripple axis (Case 4). (a) Photo of experimental set-up in wave basin at NUS with arrow indicating mainstream direction $(x)$ of current (b) Semi-log plot of typical mainstream velocity profiles; only data within $z$-range indicated by dashed lines used to obtain best fit logprofile. 
the current, which is parallel to the ripple crests. One might therefore expect that the current, just as found for the case of current alone in the direction parallel to the ripple crests, would not experience any ripple-related roughness. This anticipation is obviously incorrect since (4) shows that the bottom roughness for combined wave-current flows far exceeds that of (2) for currents alone, $1.58 \mathrm{~cm}>>$ $0.025 \mathrm{~cm}$. One possible reason for this difference in bottom roughness in the presence and absence of waves for our strictly $2 \mathrm{D}$ ripples could be that the current direction is not exactly parallel to the ripple crests when waves are present. In the closed basin the mass transport associated with progressive waves gives rise to a return current in the direction opposite of the wave propagation, i.e. perpendicular to the direction of the current introduced into the wave basin. The existence of this wave-associated current is clearly present in our experiments, as seen in Figure $3 \mathrm{~b}$ which shows the 3 minute average current component, $v$, in the direction opposite of wave propagation. For clarity, the plot of transverse velocities is linear rather than semi-log, and shows the measured velocities in the presence and absence of waves. Clearly, the transverse velocity in the presence of waves is quite substantial and virtually nil in the absence of waves. A simple calculation of the second-order return current for the given wave conditions gives a wave-associated return current of $V_{r}=1.8 \mathrm{~cm} / \mathrm{s}$, in reasonable agreement with the measured transverse velocities, which are also affected by boundary layer streaming. With a current velocity of $\sim 13.9 \mathrm{~cm} / \mathrm{s}$ in the direction parallel to the $2 \mathrm{D}$ ripples, this suggests that the actual current in the presence of waves is at an angle of roughly $7.5^{\circ}$ to the ripple axis, i.e. not "exactly" parallel to the ripple axis. Although we are unable to account quantitatively for this slight misalignment of the current relative to the ripple axis in the presence of waves, we believe that this may be a contributing factor to the relatively large roughness obtained in our combined wave-current experiments.

\section{Case 4: Current alone at $30^{\circ}$ to ripples}

The present set-up in the wave basin at the Hydraulics Laboratory at NUS to accommodate the study of combined wave-current flows is limited to currents that are "nominally" perpendicular to the waves. As discussed in the previous section (Case 3) this set-up does not produce strictly orthogonal wave-current flows. Due to the wave-induced mean flow, the resulting current is at a slight angle (of the order $10^{\circ}$ ) to the direction of the ripple axis. To investigate the near-bottom flow characteristics of currents at an angle to the ripple axis, the artificial ripples were installed at an angle of $30^{\circ}$ to the current channel direction, as shown in Figure 4a. Figure 4a shows, in addition to the placement of the artificial ripples, also "wave-guides" placed perpendicular to the ripple axis. These wave-guides were installed in anticipation of future experiments involving combined wave-current flows with the current channel rotated $30^{\circ}$ in the counter-clockwise direction, and generating waves perpendicular to the ripples and a nominal current-direction at $30^{\circ}$ to the ripple axis.

For this case current velocity profiles were again measured within the region of near-uniform flow. At each location, measurements were made over a ripple crest and the two adjacent ripple troughs. A typical semi-log plot of the current velocity component, $u_{x}$, in the mainstream direction, i.e. in the direction of the centerline of the current channel $(x)$, is shown in Figure $4 \mathrm{~b}$. Log-profile analysis at $\sim 40$ such profiles produces an experimental value for the roughness experienced by the current in the mainstream direction

$$
k_{N c x}=2.0 \pm 1.1 \mathrm{~cm}
$$

It is encouraging to notice that this value falls between the roughness values of $18.7 \mathrm{~cm}$ and 0.025 $\mathrm{cm}$, obtained for perpendicular and parallel current directions relative to the direction of the ripples. Thus, our experiments conclusively demonstrate the reality of a direction-dependent roughness for current flows over strictly 2D ripples, in agreement with the physical argument presented by Barrantes and Madsen (2000) and their experimental results.

Following Barrantes and Madsen (2000), we resolve the measured current velocity vector,

$\left(u=U_{x}, v\right)$, into its components perpendicular and parallel to the ripple axis, $U_{\perp}$ and $U_{\|}$, respectively. Typical semi-log plots of the profiles of $U_{\perp}$ and $U_{\|}$are shown in Figures $5 \mathrm{a}$ and $5 \mathrm{~b}$, respectively, and carrying out log-profile analyses for some 40 profiles we obtain the corresponding bottom roughness value,

$$
\begin{aligned}
& k_{N c \perp}=15.5 \pm 4.0 \mathrm{~cm} \\
& k_{N c \|}=0.70 \pm 0.44 \mathrm{~cm}
\end{aligned}
$$



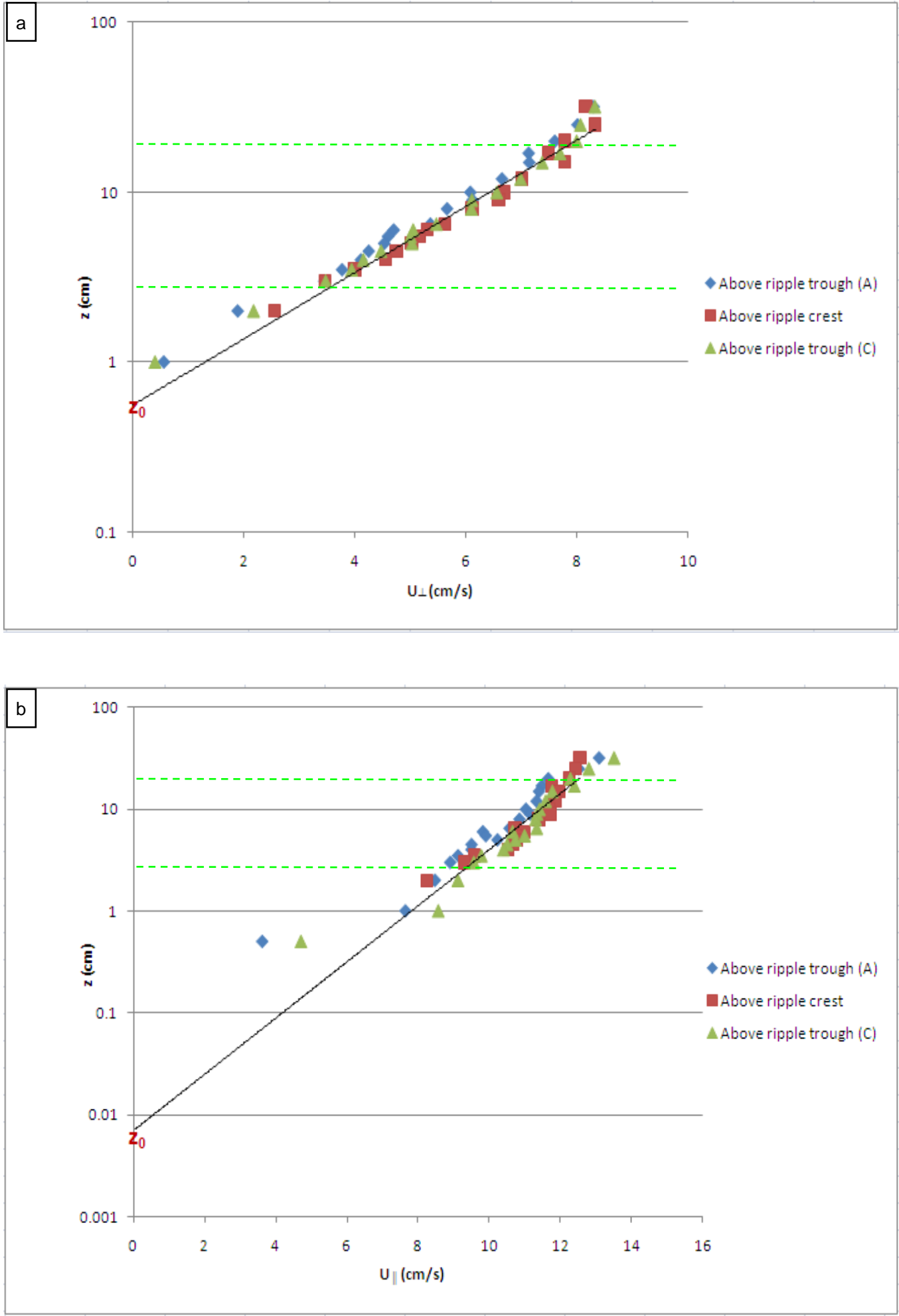

Figure 5. Typical semi-log plots of current velocity profiles for current at $30^{\circ}$ to ripple axis (Case 4). (a) Velocity component perpendicular to ripples (b) Velocity component parallel to ripples. In both (a) and (b) only data within z-range indicated by dashed lines used to obtain best fit log-profile. 

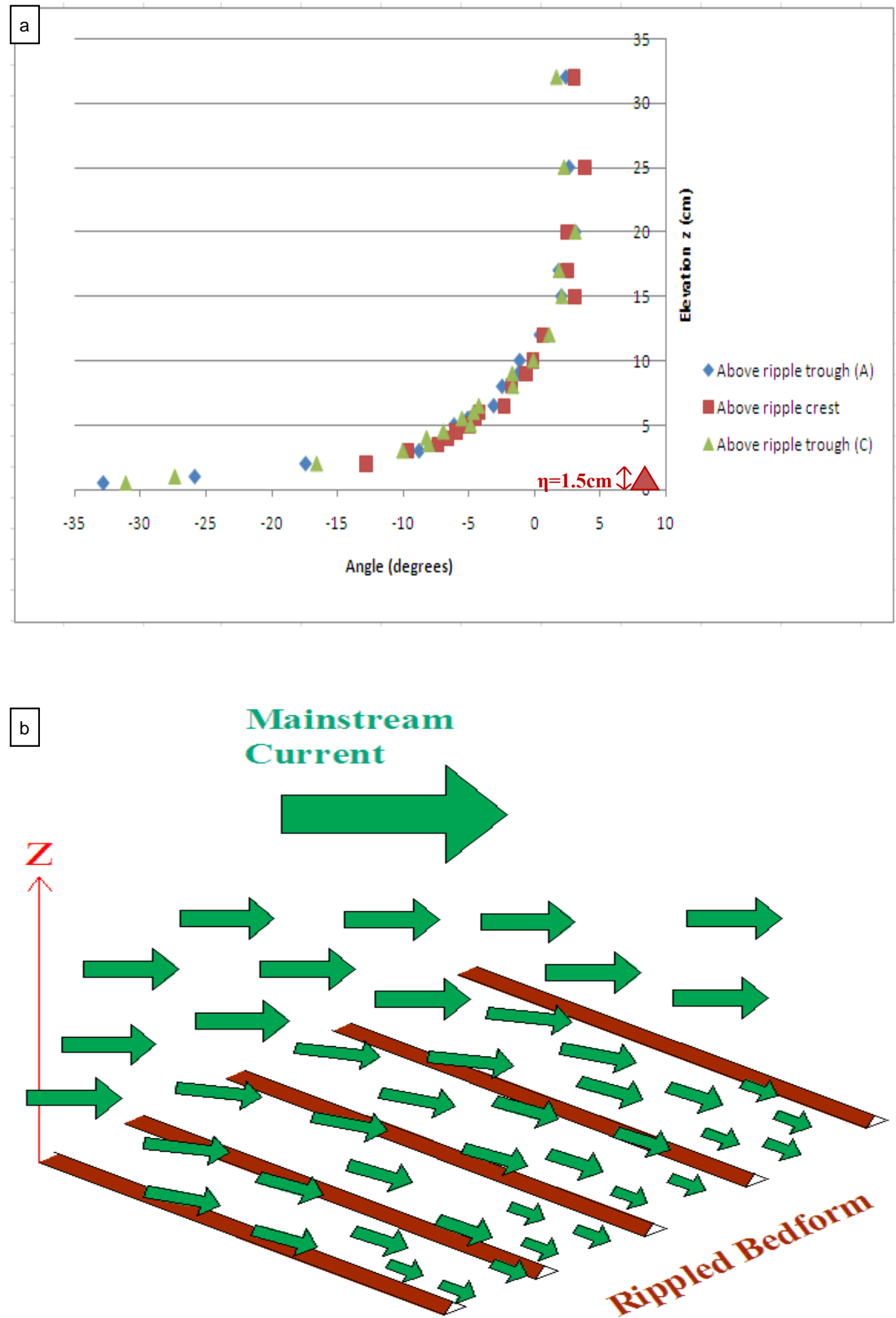

Figure 6. Turning of current vector for currents at $30^{\circ}$ to ripple axis (Case 4). (a) Velocity vector angle with mainstream direction (b) Illustration of the topography-induced near-bottom flow field for currents at "small" angles to 2D ripples. 
It should be pointed out that there is no theoretical justification for the assumption of the validity of the $\log$-profile describing the variation of $U_{\perp}$ and $U_{\|}$(or $U_{x}$ for that matter). However, the resulting roughness estimate for the component perpendicular to the ripples, given by (7), is in remarkable agreement with the roughness obtained for strictly perpendicular currents, $18.7 \mathrm{~cm}$ as given by (1), and this may form the basis for a future attempt at establishing a semi-theoretical model for this type of flow.

Unfortunately, we are unable to explain the value obtained for the roughness experienced by the parallel velocity component. All we can say is that its magnitude, being far greater than the value obtained for strictly parallel currents, suggests some kind of interaction between the turbulence generated by the flow perpendicular to the ripples and the velocity parallel to the ripples. Such an interaction may also be involved in creating the large roughness experienced by the ripple-parallel current component in the presence of near-perpendicular waves obtained for Case 3.

The nature of the physical characteristics of the near-bottom flow of a current at an angle to the axis of 2D ripples is illustrated by the plot (Figure 6a) of current vector angle relative to the mainstream $(x)$ direction. For $z>\sim 10 \mathrm{~cm}$ the current is closely aligned with the mainstream, i.e. the current channel, direction. The slight deviation from being "exactly" in the mainstream direction could be either imperfect alignment of the current meter axis with the $x$-direction or evidence of a counter flow needed to balance the strong lateral $(y)$ flow associated with the turning of the velocity vector towards the direction of the ripple crests (at $30^{\circ}$ to mainstream direction) as the bottom is approached from above, i.e. for $z<10 \mathrm{~cm}$. A more visually pleasing illustration of this topographically-induced turning of the current towards the direction of the ripples (in fact, becoming "exactly" parallel to the ripples in the troughs between ripple crests where $z<\eta=1.5 \mathrm{~cm}$ ) is shown in Figure $6 \mathrm{~b}$.

\section{CONCLUSIONS}

Through a series of carefully conducted experiments in the Hydraulics Laboratory at the National University of Singapore involving currents and combined wave-current flows over a bottom covered by artificial 2D ripples (made from $1.5 \mathrm{~cm}$ high right-angle aluminum profiles, spaced at $10 \mathrm{~cm}$ intervals) we have shown that

- The bottom roughness experienced by a current parallel to the ripples increase dramatically (from $0.025 \mathrm{~cm}$ to $1.58 \mathrm{~cm}$ ) in the presence of waves, but does not approach the value $(18.7 \mathrm{~cm})$ obtained for currents perpendicular to the ripples, as suggested by the movable bed experiments presented by Madsen et al. (2008).

- Currents alone experience a bottom roughness that increases with angle between mainstream current and ripple axis direction from $0.025 \mathrm{~cm}\left(0^{\circ}\right)$, to $2.0 \mathrm{~cm}\left(30^{\circ}\right)$, to $18.7 \mathrm{~cm}\left(90^{\circ}\right)$, i.e. definite experimental evidence of a direction-dependent roughness.

- The current velocity component in the direction parallel to the ripples experiences a bottom roughness that increases with increasing angle between mainstream flow and ripple axis directions, $0.025 \mathrm{~cm}\left(0^{\circ}\right)$ to $0.70 \mathrm{~cm}\left(30^{\circ}\right)$. A possible reason for this increase may be an interaction of the parallel velocity component and turbulence generated by the flow perpendicular to the ripples. This conjecture also supports the finding of a dramatic increase in roughness experienced by a ripple-parallel current when near-perpendicular waves are present.

- The current velocity component in the direction perpendicular to the ripple axis experiences the same bottom roughness regardless of angle between the mainstream current and ripple axis directions.

- For angles less than $30^{\circ}$ between mainstream current and ripple axis directions, and most likely also for somewhat larger angles, the current vector turns towards the direction of the ripple crests as the bottom is approached from above.

It is important to emphasize that the above conclusions are based on experiments conducted with artificial, and therefore strictly 2D, ripples covering the bottom. The extent to which some of these conclusions hold, also when the ripples are natural wave-generated movable bed ripples, remains to be seen. However, even if only partially valid for movable bed conditions when waves are present, the observed turning of the current vector as the bottom is approached from above may have significant effects on the magnitude and certainly on the direction of the net sediment transport caused by the combined action of waves and currents in coastal waters. 


\section{ACKNOWLEDGMENTS}

The authors gratefully acknowledge the financial support of the National Research Foundation of Singapore (NRF) through the Singapore-MIT Alliance for Research and Technology's (SMART) Center for Environmental Sensing and Modelling (CENSAM) program.

\section{REFERENCES}

Barrantes, A.I., O.S. Madsen. 2000. Near-bottom flow and flow resistance for currents obliquely incident to two-dimensional roughness elements. Journal of Geophysical Research 105(C11), 26, 253-264.

Madsen, O.S. 1994. Spectral wave-current bottom boundary layer flows. Proceedings 24th International Conference on Coastal Engineering, ASCE, Kobe 1, 384-398.

Madsen, O.S., S. Kularatne, H.F. Cheong. 2008. Experiments on bottom roughness experienced by currents perpendicular to waves. Proceedings $31^{\text {st }}$ International Conference on Coastal Engineering, ASCE, Hamburg, September 2008, 1, 845-853.

Mathisen, P.P., O.S. Madsen. 1996. Waves and currents over a fixed rippled bed: II. Bottom and apparent roughness experienced by currents. J. Geophysical Research 101(C7), 16, 543-550.

Negara, A.S. 2009. Experimental study of turbulent current over fixed $2 D$ and $3 D$ bottom roughness. Thesis submitted for the degree of Master of Engineering, Department of Civil Engineering, National University of Singapore.

Schlichting, H. 1960. Boundary Layer Theory, fourth edition. McGraw Hill, New York. 